\title{
Poder, teoría queer y cuerpo Cyborg
}

\author{
Power, queer theory and Cyborg body
}

DANIEL PERES DÍAZ*

\begin{abstract}
Resumen: En el presente artículo se analizan los conceptos de poder, género, sexo, cuerpo y Cyborg a partir de la ligazón entre teoría queer, ciberfeminismo y una ontología que podría calificarse como "posthumanista". El objetivo principal radica en deconstruir el discurso del poder hegemónico y dibujar las líneas maestras de un nuevo proyecto emancipatorio basado en la implementación de tecnologías inteligentes que mejorarían las capacidades del ser humano y darían forma a un sujeto nómada, cuya peculiar condición ontológica le convertiría en un ser subversivo. Para ello, se propone una crítica de los conceptos mencionados como hilo conductor de la argumentación.

Palabras clave: Poder, teoría queer, cuerpo, ontología Cyborg, posthumanismo.
\end{abstract}

\begin{abstract}
In this article discusses the concepts of power, gender, sex, body and Cyborg from the nexus between queer theory, cyberfeminism and an ontology that could be described as "posthumanist". The main objective is to deconstruct the discourse of hegemonic power and draw the outlines of a new emancipatory project based on the implementation of intelligent technologies that enhance the capabilities of human beings and they would shape a nomadic subject, whose peculiar ontological status make him a subversive being. To this purpose, it is proposed a critique of the concepts above as common thread of the argument.
\end{abstract}

Keywords: Power, queer theory, body, Cyborg ontology, posthumanism.

\section{Introducción}

En el momento actual de revolución de las biotecnologías, tecnologías de la información, psicofarmacología y ciencias cognitivas, la crítica del sistema sexo-género se imbrica necesariamente con una reflexión acerca de las posibilidades reales de transformar la realidad óntica del cuerpo en un proyecto más amplio e integrador de "mejora humana". Ello nos

Fecha de recepción: 29/05/2016. Fecha de aceptación: 20/07/2016.

* Becario de colaboración en la Universidad de Granada. Líneas de investigación: teoría queer, género, populismo, economía política del desarrollo. Dos publicaciones recientes: Peres Díaz, D. (2015) «La construcción del en-común político: Hegemonía, disputa por el sentido e hiperpolítica», en Pensamiento al margen. Revista digital, $\mathrm{n}^{\mathrm{o}}$ 3, pp. 1-16; y Peres Díaz, D. (2016) «Los límites de lo humano: Teoría queer, ciberfeminismo y ontología Cyborg», Tales. Revista de la Asociación de estudiantes de Filosofía, nº6, pp. 151-162. Correo electrónico institucional: peres@correo.ugr.es 
constriñe a emprender una serie de deliberaciones con importantes implicaciones ontológicas, epistemológicas, éticas y políticas, centrándonos especialmente en el advenimiento y consolidación de nuevas subjetividades.

Frente a la idea del cuerpo como límite negativo a la apertura de nuevas realidades, puede vislumbrarse un nuevo marco epistémico que opera en la frontera, en las diversas posibilidades de transformar, manipular, cambiar o hacer del cuerpo una entidad abierta, susceptible de recibir nuevos significados y, en línea con ello, de generar diferentes espacios de pensamiento. Entonces, es posible articular, dar forma, a una noción positiva de límite cuya condición de posibilidad actual es el surgimiento de las nuevas tecnologías, y rastrear a partir de ella algunas señas que podrían hacernos pensar en un humanismo biotecnológico entendido como un nuevo proyecto posthumanista.

En esta línea, considero que el concepto de "cuerpo Cyborg" es el soporte idóneo para materializar dicho humanismo, pues constituye un espacio genuino de reflexión que engloba la relación entre cultura y naturaleza en toda su amplitud, al tiempo que perfila una plataforma adecuada para pivotar la transición hacia una nueva ontología en la cual la disolución de las oposiciones actúa como una fuerza política subversiva.

Así, retomando la famosa frase de Stelarc, podemos decir que "el cuerpo está obsoleto", lo que significa que este debe ser reconceptualizado, resignificado. Dado que el cuerpo, entendido como una entidad natural, es insuficiente para describir en términos ontológicamente adecuados los retos, intelectuales y políticos, a los que inexorablemente debemos hacer frente hoy, se propone una visión alternativa según la cual el poder es generador de realidades corpóreas, ónticas, que desembocan en una hegemonía cultural y simbólica. Y puesto que, desde las posibilidades tecnológicas, se pueden deconstruir el sexo y el género, es posible que emerja un Cyborg que es en sí mismo un sujeto subversivo.

Así las cosas, en la exposición se enlazan las propuestas de Judith Butler y la teoría queer con la idea del cuerpo Cyborg. El análisis de Butler nos va a servir de punto de partida para luego esbozar la propuesta de una ontología posthumanista, fundada en la idea de un Cyborg, en conexión con el ciberfeminismo de Haraway. El hilo conductor, por tanto, anida en un enfoque y metodología "deconstructivista", en la medida en que se busca deconstruir las categorías fundamentales que son objeto de examen en el presente trabajo.

\section{Poder en el cuerpo: La teoría queer}

La construcción contemporánea del cuerpo está claramente sobrepasada por la visión tradicional del poder según la cual este se trasmite de un modo vertical, fácilmente identificable y a través de instituciones más o menos visibles. Más bien, la problemática del cuerpo, su construcción social y científica, pone de manifiesto un poder de una naturaleza distinta, un poder disperso, difuso, que se expande subrepticiamente y se consolida gracias a prácticas sociales e instituciones aparentemente neutras cuyo objetivo último es la legitimación de un orden cultural, político y económico dominante (Peres Díaz, 2015).

Por ello, la crítica del cuerpo ha de ser una crítica biopolítica, esto es, debe centrarse en examinar los mecanismos de construcción socio-cultural del cuerpo más que en las instituciones que operan "visiblemente" en él. De ello se infiere una actitud crítica por defecto, ya que es en ese acervo de biopoder, en esos dispositivos, donde anida el verdadero vector 
creador de realidades. Así pues, el primer paso lógico que debe darse es una deconstrucción del imaginario colectivo occidental, haciendo un énfasis especial en la relación entre cuerpo, poder y sexo-género, ya que ello nos permitirá enlazar con la teoría queer. Como afirman Madera Pacheco y Pacheco Ladrón de Guevara:

La cultura occidental se basa en el dualismo jerarquizante y antagónico que afecta a todos los ámbitos del conocimiento, de la cultura y de la ética y lo establece como formas masculinas de relación. Esas formas masculinas de relación se desarrollan en la exclusión del mundo de las mujeres y de las formas de vida no humana. Se convierten, a su vez, en arquetipos profundos de la cultura, alimentados por sistemas de creencias, valores y actitudes, uno de ellos es la construcción de las mujeres como cuerpo-sexo, y su reducción a naturaleza. $(2015,57)$

Como puede constatarse, el enemigo a batir es el esencialismo y el pensamiento binario. La crítica al binarismo y al pensamiento oposicional en términos de categorías lo encontramos de un modo claro y conciso en el planteamiento de Butler (2007), cuya tesis nuclear viene a decir que el género es el producto de un complejo proceso de construcción social y cultural. En esta línea, se sostiene que el sexo-género se construye discursivamente, esto es, lingüística y culturalmente. No habría, por tanto, un sexo natural, material y prediscursivo (Butler, 2002), sino tan solo su significación social y cultural en tanto que género.

El género, pues, no es sino una construcción que alcanza forma a partir de la repetición de unas prácticas cotidianas incrustadas en la estructura de la sociedad. De este modo, el género no existe sino en la performatividad del lenguaje, es decir, solo tiene sentido y es comprensible desde el momento en que lingüísticamente lo designamos (Pérez Navarro, 2004). En consecuencia, el género no es algo que preexiste al lenguaje, sino que se crea en el lenguaje. A juicio de Butler, el género no es algo "sustantivo", sino, como digo, una construcción performativa, algo que "constituye la identidad que se supone que es" (Butler, 2007, 58).

Dando un paso más, podemos afirmar que el sujeto depende de una regla discursiva, esto es, existe gracias a que tenemos un nombre para decir que existe. En otras palabras: "algo que no es sujeto deviene sujeto gracias a la reglamentación discursiva" (Butler, 2006, 83). Así las cosas, el acto de nombrar resulta ser no ya un mero acto de designación, que haría referencia a una conexión conceptual entre dos entidades previamente conocidas, sino la inclusión en el lenguaje del momento fundamental que constituye la cosa misma, o sea, la creación instantánea de una realidad concreta (Argañaraz, 2012). Cuando nombramos, pues, estamos inscribiendo realidades indeterminadas en unas lógicas culturales, simbólicas, económicas y políticas muy concretas.

Estas lógicas cohabitan, se superponen y ensamblan una hegemonía, entendiendo por esta un proceso de repetición de prácticas sociales que refuerzan determinados ideales y representaciones colectivas sobre cómo ha de organizarse la vida, el trabajo, la reproducción, la economía, la ciencia, la identidad, la sexualidad, etc. Con ello, se establece una biopolítica en el sentido de una creación de actitudes vitales, de disposiciones aprehendidas que constituyen un a priori conductual para sí y para con los demás. Y luego, esa biopolítica genera exclusiones jerarquizantes (Romero, 2003). 
Si esto es así, o sea, si el sexo-género se construye discursivamente, significa entonces que el control de la subjetividad responde a una "gramática de la gobernabilidad". En efecto, si entendemos que el lenguaje es capaz de construir realidades nuevas que no existían previamente, parece como mínimo razonable pensar que quienes detentan el poder de cambiar, manipular, transformar o imponer un lenguaje tienen, en última instancia, el poder de controlar los cuerpos (Peres Díaz, 2016a).

Por lo tanto, estaríamos ante un modo de legitimación de unas formas de estar en el mundo y, sensu contrario, ante una manera de discriminación e invisibilización de otras:

(...) Se produce un "cierre categorial" cuyo fin último anida en excluir toda forma de subjetividad que cuestione la validez del orden social hegemónico. Ese cierre nos obliga a entender el género como un dispositivo de poder, esto es, como un operador que regula la vida social en interacción con otros dispositivos. Desde un análisis foucaultiano, podríamos decir que el género está construido sobre la base de unas relaciones de poder dispuestas de un modo reticular, y destinadas a generar un poder microfísico que legitima unas prácticas al tiempo que invisibiliza otras (Peres Díaz, 2015, 658).

El poder, en este marco, se manifiesta a través de criterios de veridicción, de patrones de demarcación entre lo bueno y lo malo, lo correcto y lo incorrecto, etc. Por eso, el poder es en y a través del cuerpo, ya que, de este modo, se interioriza en él una serie de vivencias que son las que posibilitan designar estados de ánimo, formas de conducta o identidades. La validación del "modo apropiado" de comportarse se produce mediante una sanción social, generalmente lingüística o simbólica, que indica que "estamos haciendo lo correcto".

Es por todo ello que la visibilización de sexualidades, y más genéricamente identidades, periféricas, esto es, que bordean la norma, es por su mera existencia una herramienta cuestionadora del régimen y del poder hegemónico (Fonseca Hernández, \& Quintero Soto, 2009). El caso de Herculine Barbin analizado por Foucault (2007) es un claro ejemplo de esto; Herculine, como hermafrodita, cuestionaba el orden legal en tanto en cuanto no era ni hombre ni mujer, sino ambos y al mismo tiempo ${ }^{1}$. Se observa el carácter coactivo del Derecho en la genealogía de la sexualidad que emprende Foucault (1998), donde se muestra la clara arbitrariedad del sexo-género, demostrándose así que todo concepto tiene una génesis y una evolución destinadas a legitimar un estado de cosas. Se trata de narrativizaciones, ficcionalizaciones del pasado orientadas a validar un poder que se constituye en hegemónico (Suárez Briones, 1999). Más claramente, la imposición de una perspectiva clínica de conductas sexuales "marginales", como la transexualidad, es producto de una situación ideológica y tecnológica concreta (Balza, 2009).

Ahora bien, si somos capaces de relativizar el género, y con él todas las categorías que nos son dadas, aplicando el método genealógico foucaultiano, podremos poner de relieve la

1 Lo mismo ocurre con la transexualidad, cuya despatologización médica no ha venido acompañada en la práctica de una crítica de los roles de género. La verdad es que las personas transexuales tienen dificultades para encontrar coordenadas simbólicas que les permitan interpretar su propio cuerpo desde el momento en que la sociedad sigue imputando sexos y géneros unívocos y binarios (Rubio Arribas, 2009). 
arbitrariedad de nuestros propios conceptos y, con ello, abonar el campo para la apertura de nuevas subjetividades (Córdoba García, 2003).

En este contexto, la teoría queer aboga por la proliferación de nuevas identidades que rebasen el marco y que permitan crear una brecha en la conformación y consolidación de realidades fijas, inmutables, claramente establecidas. Se trata de un "deshacer el género" (Butler, 2006) que nos lleva inmediatamente a un "deshacer las categorías". En esa tarea las posibilidades que ofrece la convergencia entre biotecnologías, tecnologías de la información y el cuerpo son, cuando menos, interesantes de cara a la discusión que se quiere inducir en el presente trabajo. Por ello, la idea de un Cyborg nos va a permitir dilucidar la cuestión de la identidad y el cuerpo desde las posibilidades que ofrece la tecnología en el marco de una nueva ontología.

El hilo conductor que permite conectar este primer apartado con el segundo estriba en el carácter abierto de la identidad y el género, como construcción cultural, en un caso, y como cuerpo Cyborg, en el otro; y en su potencial de subversión política al derrumbar los binomios de la lógica bivalente y la ontología monovalente.

\section{Superando paradigmas: El cuerpo Cyborg como nueva ontología}

La convergencia entre nanotecnologías, biotecnologías, tecnologías de la información y ciencias cognitivas (tecnologías NBIC) puede alcanzar en el futuro un estado de desarrollo lo suficientemente avanzado como para pensar que es posible su uso fiable con fines de rediseño y mejora de la especie humana. De este modo, hay autores que plantean como plausibles aplicaciones tales como la creación de fármacos génicamente especializados, la inserción de genes con rasgos favorables, la clonación y reemplazo de órganos, el diagnóstico preimplantarorio, la eugenesia (positiva y negativa), la modulación del comportamiento y de la respuesta fisiológica, la mejora de las capacidades cognitivas, funcionales, metabólicas o inmunológicas, la integración de dispositivos auxiliares para procesar e interpretar información masiva, la prolongación de las expectativas de vida y el retraso del envejecimiento o la conexión directa del cerebro humano a una base de datos infinita.

Ante estos retos, la filosofía debe dar una respuesta que abarque las implicaciones onto-epistémicas, pero también éticas, sociales y políticas, en un marco teórico que supere la dicotomización entre transhumanistas y bioconservadores, por ser posturas enteramente reduccionistas, esencialistas y simplificadores del debate en cuestión, y adoptando un enfoque que yo denomino "posthumanista".

El posthumanismo, en conexión con diferentes estudios de prospectiva científica y tecnológica de carácter interdisciplinar, viene a sostener que estamos muy cerca de conseguir las herramientas que harán posible reducir de manera radical las limitaciones tradicionalmente asociadas a la especie humana en su condición natural, abriendo de este modo un horizonte que justificaría hablar de individuos posthumanos, claramente diferenciados en sus capacidades de los humanos no rediseñados, pero sin querer por ello eliminar el cuerpo y la historia como elementos transversales de lo que somos y nos constituye, como sí que pretende el transhumanismo, o postular una esencia natural, como sostiene el enfoque bioconservador, que se muestra a todas luces insatisfactorio para describir en términos epistemológicamente adecuados el potencial de las tecnologías NBIC. 
La era del ciberespacio, en la que nos hallamos irremediablemente insertos, como se deduce de la hiperexpresión en que se ha convertido Internet y las redes sociales, transforma la concepción del individuo y del yo; se produce de este modo una torsión del centro de gravedad de la metafísica tradicional, dependiente en exceso de una ontología monovalente incapaz en última instancia de dar cuenta del nuevo paradigma de la información. Frente a la noción de cuerpo como objeto de estudio, esto es, como una cosa tematizada por las ciencias naturales durante la Modernidad en algo meramente objetivo y mecánico (Aguilar, 2007a), la posibilidad de comunicación en esa red de interrelaciones que es el ciberespacio provoca un vaciamiento de la construcción del yo, de deconstrucción de "nuestra sustancia" en el conjunto de fuerzas y relaciones que sujetan lo que somos (Aguilar, 2012).

O sea, el yo, en este marco, es fundamentalmente posibilidad, sujeción de relaciones en constante proceso de disolución y acoplamiento. Ello permite articular un intento de deconstrucción, consistente en desedimentar las bases de la civilización occidental atacando las categorías sustancialistas de la metafísica de la presencia y posibilitando la emergencia de nuevas identidades deslocalizadas, no jerarquizantes, inclusivas y ontológicamente políticas. La apuesta del Ciberfeminismo sería precisamente la de buscar la emancipación de la mujer a través del Cyborg (Aguilar, 2007b).

En este contexto, la ontología Cyborg es una posición "intermedia" entre transhumanismo y bioconservadurismo, una ontología que aquí se caracteriza como "posthumanista" en la medida en que integra las posibilidades transformadoras de las NBIC con la realidad óntica del cuerpo. Esa posición intermedia, la ontología posthumanista, que se funda en la idea del cuerpo Cyborg, no pretende superar el yo en una alteridad totalmente otra, sino combinar el potencial de la tecnología para hacer del cuerpo una entidad más plástica y, con ello, flexibilizar al yo. Para eso, habría que ensayar un humanismo tecnológico (Molinuevo, 2004) en el que las tecnologías no fuesen elementos de alienación, sino herramientas al servicio de un ideal de humanidad focalizado en la mejora.

No en vano, ya usamos la tecnología para fines humanistas, y la educación persigue eso mismo; no habría diferencia entre lo que hacemos ahora y lo que haríamos si aplicásemos tecnologías NBIC en el futuro, siendo así que estas y la educación, que es el modo actual en que las sociedades humanas buscan la "mejora humana", convergen en un mismo fin. O sea, al usar la tecnología no estaríamos empleando herramientas muy diferentes a las que ya usamos, por lo que no tendría sentido criticar el empleo de, por ejemplo, psicofármacos o implantes neuronales para conseguir mejorar las capacidades humanas arguyendo que "eso va contra natura".

De facto, ya estamos rompiendo la barrera entre mejora y terapia humana. Cuando usamos vacunas, por ejemplo, no estamos curando a un enfermo, sino mejorando el sistema inmunológico para prevenir futuras eventualidades; del mismo modo, la terapia génica o el uso de psicofármacos va en la misma línea. No podemos apelar a una naturaleza o esencialidad humana, que previsiblemente debe ser cuidada, cuando todos los días tomamos café para mejorar nuestra percepción y atención, usamos lentes para corregir la visión y empleamos complejos vitamínicos para suplir carencias alimentarias.

En este sentido, Sloterdijk (2000) habla de "reglas para el parque humano" haciendo referencia precisamente a eso, a la idea de que el objetivo perseguido por la educación es la mejora humana, exactamente lo mismo que pretende alcanzarse mediante el uso de estas tecnologías. Por otro lado, habría que hacer una distinción capital en este punto, a saber, la 
distinción entre "alotécnicas" y "homeotécnicas" (Sloterdijk, 2004), esto es, entre técnicas que nos hacen daño, que tienen efectos secundarios y que no contribuyen a que alcancemos nuestros objetivos, y técnicas inteligentes que nos pueden ayudar a mejorar nuestras capacidades. Un ejemplo de alotécnica sería un fármaco que mejora la memoria pero lesiona el hígado, mientras que un ejemplo de "homeotécnica" sería una cápsula con nanorobots que se implementan en las células vivas para conseguir prevenir el cáncer sin ocasionar ningún tipo de lesión o daño. La confluencia entre biología molecular, cibernética y neurociencia haría posible la consolidación de estas "homeotécnicas".

Pero para que esta visión que pretende superar el reduccionismo tanto de la postura bioconservadora, que postula un sujeto hipostasiado, sin historia, como de la postura transhumanista, que en el fondo es un neoplatonismo que niega la realidad del cuerpo, consiga dotarse de eficacia es necesario deconstruir el imaginario utópico-distópico de las tecnologías.

Efectivamente, un análisis de las narrativas digitales de la segunda mitad del siglo XX nos muestra una visión totalmente anacrónica de las tecnologías. La imagen que trasmiten la literatura de ficción y el cine distópico responde a una visión determinista del ser humano en la que, para bien o para mal, las tecnologías funcionan independientemente del decurso de la historia humana, cayendo en una flagrante falacia de pendiente resbaladiza.

Matrix, Fahrenheit, 1984, Un mundo feliz o Gattaca son algunos casos que responden a esa lógica determinista y deshumanizante propia de la Nueva Izquierda tecnófoba de segunda mitad del siglo XX, la cual viene a decir que el modelo de racionalidad moderno ilustrado responde al esquema científico-técnico-industrial que termina en una cosificación del hombre. Es indudable que, ya sea en su visión utópica o distópica, este imaginario estético parte de una visión simplista en extremo de las tecnologías que obvia el impacto humano que subyace al desarrollo e implantación de las tecnologías en las que serán las futuras comunidades humanas.

Más bien, se trata de entender, tal y como hace Rifkin (2009), la biotecnología como arte, es decir, como creación, negando que la naturaleza sea un campo de restricciones y entendiéndola en su lugar como campo de transformación. El cuerpo se revela como inservible sin las prótesis tecnológicas que lo capacitan para funcionar en el mundo actual; la ampliación del cuerpo humano por el aparato tecnológico, la posibilidad de llegar a una realidad aumentada, es el nuevo reto que los sujetos de las sociedades emergentes deben plantearse como aceptación incondicional de una nueva naturaleza, más abierta desde que descubrimos la textualidad del cuerpo con el ADN a partir del Proyecto genoma humano.

Además, el Cyborg, al no responder a las categorías bivalentes tradicionales de sexo, raza, género, etc. se constituye como un nuevo sujeto revolucionario (Haraway, 1990). Por esta razón, se da una intrínseca relación entra la idea del cuerpo Cyborg y una nueva visión de la biopolítica:

Igualmente, el Cyborg, al no responder a las categorías bivalentes tradicionales, se constituye como nuevo sujeto revolucionario en tanto que no funda una identidad sobre una clase social, una etnia específica o un colectivo determinado. Por esta razón, se da una intrínseca relación entra la idea del cuerpo Cyborg y una nueva visión de la (bio)política. En efecto, un sujeto desprendido de las categorías bivalentes sería un sujeto revolucionario en tanto en cuanto la construcción de su identidad sería mucho más abierta, independiente en cierto sentido de los patrones sociales, 
lo que significa que por su propia condición estaría arrojado insoslayablemente al cuidado de sí. Liberados de gran parte de nuestra dependencia biológica, así como de los roles claros impuestos por la dinámica económica de las sociedades capitalistas avanzadas, los sujetos Cyborg deberán emprender la tarea de construir una comunidad de la diferencia, asentada no ya en una identidad sustancial -la nación, la religión, la cultura- sino sobre la apertura de todo individuo a hacerse a sí mismo. (Peres Díaz, 2016b, 159)

Estaríamos a las puertas de un sujeto fronterizo, nómada, cuya morada no sería otra que el devenir mismo, la contextura de lo real en tanto que singularidad irreductible e incardinada en una red de posibilidades fluctuantes e inagotables. El Cyborg, asentado sobre la observación de que el cuerpo importa y sobre el compromiso ontológico de que es el cuerpo el vehículo fundamental por el que transita el poder, serviría a los propósitos de una ontología inherentemente política.

Una vez rotas las barreras entre el humano y la máquina, la naturaleza y la cultura, lo dado y lo construido, estaremos en disposición de comprender el cuerpo como evento, como híbrido (Haraway, 1990). Y que el cuerpo sea un evento no quiere decir, ni más ni menos, que los cuerpos han de ser insumisos a las normas reguladoras del sexo, el género y la identidad; significa que el cuerpo, dimensión constitutiva de lo que somos y de lo que queremos ser, no tiene ninguna limitación natural a priori y fuera del discurso. Por consiguiente, es en las posibilidades actuales del discurso en que se cifra el proyecto individual y colectivo de las no-identidades humanas.

En última instancia, se trataría de suprimir el cuerpo físico, orgánico, y adentrarnos en la condición ontológica posmoderna (Lyotard, 2007), que consiste en difuminar las dicotomías por medio del concepto de lo virtual y la mezcla con la tecnología. Hecho esto, nos habríamos adentrado de lleno en una ontología posthumanista cuyo núcleo principal sería la transformación del cuerpo en Cyborg.

\section{Algunas conclusiones}

Se ha querido exponer una crítica de las nociones de poder, sexo, género, cuerpo y ontología Cyborg partiendo de la deconstrucción que opera en la teoría queer y enlazando esta con las posibilidades de transformación que ofrece la confluencia de tecnologías nanobio-info-cognitivas.

En esta línea, se ha argumentado a favor de una concepción reticular del poder, que opera en y desde los cuerpos, para proponer después la tesis de que la transformación del cuerpo permite la apertura de nuevas subjetividades, así como perfilar una ontología no bivalente que hemos denominado "posthumanista". La noción de Cyborg, como híbrido, se muestra aquí como fundamental, pues desde ella se puede repensar el marco de la ontología y las implicaciones que tendría un proyecto de estas características.

Un trabajo de este tipo en ningún caso puede ser más que una aproximación, sin ánimo de querer llevar a cabo un análisis pormenorizado, cuyo único objetivo es el de bosquejar un marco teórico lo suficientemente rico como para ir profundizando luego en aspectos más concretos y problemáticas que podrían ir surgiendo al hilo de lo planteado aquí. 
Cada dimensión o nivel de discusión es susceptible de abarcar problemas de diferente índole, como puede ser el político (¿qué valores políticos guiarán tal transformación?), económicos (¿tendría acceso todo el mundo a dicha tecnología?), éticos (¿es lícito usar la tecnología para mejorarnos?), sociales (¿está la sociedad preparada para cambiar su forma de entender el mundo?), ontológicos (¿seguiríamos siendo "humanos"?), epistemológicos (¿cómo podríamos saber lo que está por venir?), etc.

Como es obvio, quien escribe esto no puede ni pretende contestar a todas esas preguntas, sino tan solo problematizar y dilucidar, a veces intentando afectar al lector con aseveraciones bastante contundentes, para señalar o llamar la atención sobre un problema filosófico de primer orden, a mi juicio, apasionante. A tal fin, el presente artículo no es sino una modesta contribución para enriquecer un debate con cada vez más presencia y que camina inexorable en busca de respuestas.

\section{Referencias}

Aguilar, T. (2007a), «Biopolítica y fenomenología: Consideraciones en torno al cuerpo objeto», Revista Laguna, $\mathrm{n}^{\circ}$ 21, pp. 29-42. Recuperado de: http://publica.webs.ull.es/ upload/REV\%20LAGUNA/21\%20\%202007/02\%20(Teresa\%20Aguilar).pdf

Aguilar, T. (2007b), «Ciberfeminismo y ecofeminismo», Germinal, n 3, pp. 73-81. Recuperado de: dialnet.unirioja.es/descarga/articulo/2729580.pdf

Aguilar, T. (2012), Ontología Cyborg. El cuerpo en la sociedad tecnológica, Barcelona, Gedisa.

Argarañaz, C. (2012), «Una aproximación a la lógica subjetiva de la Modernidad líquida: el caso de las minorías sexuales», Revista Latinoamericana de Estudios sobre el Cuerpo, Emociones y Sociedad, $\mathrm{n}^{\circ}$ 7, pp. 43-51. Recuperado de: http://www.relaces.com.ar/index. php/relaces/article/viewFile/70/104

Balza, I. (2009), «Bioética de los cuerpos sexuados: transexualidad, intersexualidad y transgenerismo», Isegoría. Revista de Filosofía Moral y Política, no 40, pp. 245-248.

Butler, J. (2002), Cuerpos que importan: sobres los límites materiales y discursivos del sexo, Buenos Aires, Paidós.

Butler, J. (2006), Deshacer el género, Barcelona, Paidós.

Butler, J. (2007), El género en disputa. El feminismo y la subversión de la identidad, Barcelona, Espasa.

Córdoba, D. (2003), «Identidad sexual y performatividad», Athenea Digital, nº 4, pp. 87-96. Recuperado de: http://www.hartza.com/david.pdf

Fonseca, C. \& Quintero, M.L. (2009), «La Teoría Queer: la deconstrucción de las sexualidades periféricas», Sociológica, $\mathrm{n}^{\circ}$ 69, pp. 43-60. Recuperado de: http://www.revistasociologica.com.mx/pdf/6903.pdf

Foucault, M. (1998), Historia de la sexualidad (Vol. I), México D.F., Siglo XXI.

Foucault, M. (2007), Herculine Barbin llamada Alexina B., Madrid, Talasa.

Haraway, D. (1990), «Las promesas de los monstruos: una política regeneradora para otros inapropiados/bles», Política y sociedad, $\mathrm{n}^{\circ} 30$, pp. 121-163. Recuperado de: http:// dialnet.unirioja.es/servlet/articulo?codigo $=154534$

Haraway, D. (1991), Ciencia, Cyborgs y mujeres. La reinvención de la naturaleza, Madrid, Cátedra. 
Lugo-Márquez, S. (2003), «Cuerpo-artefacto: Aportes de las perspectivas de género y queer a la deconstrucción de los cuerpos 'naturalizados'», Revista Trilogía, no 9, pp. 37-46. Recuperado de: dialnet.unirioja.es/descarga/articulo/4521397.pdf

Lyotard, F. (2006), La condición posmoderna, Madrid, Cátedra.

Madera Pacheco, J.A. \& Pacheco Ladrón de Guevara, L.C. (coord.) (2015), «Miradas actuales a las ciencias sociales», Noésis. Revista de Ciencias Sociales y Humanidades, núm. especial, pp. 54-64.

Martínez-Guzmán, A. \& Montenegro, M. (2010), «Narrativas en torno al trastorno de identidad sexual», Prisma social. Revista de ciencias sociales, $n^{\circ} 4$, pp. 1-44.

Molinuevo, J.L. (2004), Humanismo y nuevas tecnologías, Madrid, Alianza.

Moral Eusebio, E. (2014), «¿Es el sexo al género lo que la naturaleza a la cultura? Una aproximación queer para el análisis arqueológico», ArqueoWeb, no 15, pp. 248-269. Recuperado de: http://pendientedemigracion.ucm.es/info/arqueoweb/pdf/15/Moral.pdf

Peres Díaz, D. (2015), «Subjetividad y legitimación: La construcción contemporánea del cuerpo de las mujeres», en: Cabrera Espinosa, M. \& López Cordero, J.A., (eds.) VII Congreso Virtual sobre Historia de las Mujeres, pp. 653-664. Recuperado de: https:// dialnet.unirioja.es/servlet/articulo?codigo $=5346961$

Peres Díaz, D. (2016a), «El género como categoría crítica: Implicaciones onto-epistémicas de la teoría queer», CuadrantePhi. Revista estudiantil de Filosofía, núm. de enero de 2016. Recuperado de: https://cuadrantephi.com/2016/01/22/el-genero-como-categoriacritica-implicaciones-onto-epistemicas-de-la-teoria-queer

Peres Díaz, D. (2016b), «Los límites de lo humano: Teoría queer, ciberfeminismo y ontología Cyborg», Tales. Revista de la Asociación de estudiantes de Filosofía, nº, pp. 151-162. Recuperado de: https://asociaciontales.wordpress.com/numero-6/

Pérez Navarro, P. (2004), «Performatividad y subversión de la identidad: a propósito de la obra de Judith Butler», Revista Laguna, n¹4, pp. 147-164. Recuperado de: http:// publica.webs.ull.es/upload/REV\%20LAGUNA/14\%20\%202004/08\%20(Pablo\%20 P\%C3\%A9rez\%20Navarro).pdf

Rifkin, J. (2009), El siglo de la biotecnología: el comercio genético y el nacimiento de un mundo feliz, Barcelona, Paidós.

Romero, C. (2003), «De diferencias, jerarquizaciones excluyentes, y materialización de lo cultural. Una aproximación a la precariedad desde el feminismo y la teoría queer», Cuadernos de Relaciones Laborales, Vol. 21, n 1, pp. 33-60. Recuperado de: http://dialnet. unirioja.es/servlet/articulo?codigo $=821197$

Rubio Arribas, J. (2009), «Aspectos sociológicos de la transexualidad», Nómadas: revista crítica de Ciencias Sociales y Jurídicas, Vol. 21, $\mathrm{n}^{\circ}$ 1. Recuperado de: http://revistas. ucm.es/index.php/NOMA/article/view/NOMA0909140361A/26234

Sloterdijk, P. (2000), Reglas para el parque humano, Barcelona, Siruela.

Sloterdijk, P. (2004), «El hombre auto-operable. Notas sobre el estado ético de la tecnología génica», Laguna: Revista de Filosofía, n 14, pp. 9-22. Recuperado de: http://dialnet. unirioja.es/servlet/articulo?codigo=1059747

Suárez, B. (1999), «Sobre hombres y márgenes. Relaciones entre feminismo y teoría queer», Lectora, ${ }^{\circ}$ 4, pp. 83-91. Recuperado de: http://dialnet.unirioja.es/servlet/ articulo? codigo $=2227633$ 